\title{
The Cauchy Problem for the Generalized Fractional Second Order Semilinear Evolution Equation ${ }^{1}$
}

\author{
Félix Pedro Q. Gómez ${ }^{2}$ \\ DAMAT-CT, UTFPR \\ Ruy Coimbra Charão ${ }^{3}$ \\ MTM, UFSC
}

\begin{abstract}
In this work we study the existence and uniqueness of solutions for a second order semilinear $\delta$ - $\alpha$ - $\gamma$-evolution equation with fractional damping term which includes some partial differential equations as plate equation under effects of rotational inertia or not and Boussinesq type that can model hydrodynamics problems.
\end{abstract}

Palavras-chave. Plate type equation. Generalized Boussinesq equation, Fractional Laplacians, Generalized rotational inertia. Fractional dissipation. Existence and uniqueness.

\section{Introduction}

We consider in this work the following Cauchy problem for a second order generalized $\delta$ - $\alpha$ - $\gamma$-evolution equation with a fractional damping in $\mathbb{R}^{n}$,

$$
\left\{\begin{array}{l}
\partial_{t}^{2} u+(-\Delta)^{\delta} \partial_{t}^{2} u+b \Delta^{2} u+a(-\Delta)^{\alpha} u+(-\Delta)^{\theta} \partial_{t} u=\beta(-\Delta)^{\gamma} u^{p}, \\
u(0, x)=u_{0}(x), \quad \partial_{t} u(0, x)=u_{1}(x),
\end{array}\right.
$$

where $u=u(t, x)$ with $(t, x) \in] 0, \infty\left[\times \mathbb{R}^{n}, b, a>0, \beta \in \mathbb{R}\right.$ constants, $p>1$ a integer and $u_{0}, u_{1}$ are the initial data. The exponents $\delta, \alpha, \theta$ and $\gamma$ of the Laplacian operator are such that $0 \leq \delta \leq 2,0 \leq \alpha \leq 2,0 \leq \theta \leq(2+\delta) / 2$ and $0 \leq \gamma \leq(2+\delta) / 2$.

\subsubsection{Abstract Semilinear Problem: Existence of Solution}

Let $X$ be a Banach space and $B$ a linear operator of $X$. Consider the abstract Cauchy problem

$$
\left\{\begin{array}{lll}
\frac{d U}{d t}=B U(t)+F U(t) & \text { for all } & t>0 \\
U(0)=U_{0} & \text { where } & U_{0} \in X
\end{array}\right.
$$

and $F$ is a function not linear.

\footnotetext{
${ }^{1}$ final

${ }^{2}$ felixgomez@utfpr.edu.br

3 charao@mtm.ufsc.br
} 
Definition 1.1. A function $F: D(B) \rightarrow D(B)$ is continuous Lipschitz in bounded sets of $D(B) \subset X$ if given a constant $M>0$ there exist a constant $L_{M}>0$ such that

$$
\|F(U)-F(W)\|_{X}+\left\|B_{1}(F(U)-F(W))\right\|_{X} \leq L_{M}\left(\|U-W\|_{X}+\left\|B_{1}(U-W)\right\|_{X}\right)
$$

for all $U$ and $W$ in $D(B)$ such that we have

$$
\|U\|_{X}+\|B U\|_{X} \leq M \quad \text { and } \quad\|W\|_{X}+\|B W\|_{X} \leq M .
$$

Theorem 1.1. Let $F: D(B) \rightarrow D(B)$ a continuous Lipschitz function in bounded sets of $D(B) \subset X$. For all $U_{0} \in D(B)$, there exist only one strong solution $U$ of Cauchy problem (2) defined in a maximal interval $\left[0, T_{m}[\right.$ such that only one of the following conditions is true
(i) $T_{m}=\infty$
(ii) $T_{m}<\infty$ and $\lim _{t \rightarrow T_{m}}\left\{\|U\|_{X}+\|B U\|_{X}\right\}=\infty$.

The solution $U$ of Cauchy problem (2) belongs to the following class

$$
U \in C^{1}\left(\left[0, T_{m}[; X) \cap C\left(\left[0, T_{m}[; D(B)) .\right.\right.\right.\right.
$$

\section{Existence and Uniqueness of Solution: Linear Problem}

Through the Theory of Semigroup we show the existence and uniqueness of solutions to the following Cauchy problem in $\mathbb{R}^{n}$ with $n \geq 1$,

$$
\left\{\begin{array}{l}
\partial_{t}^{2} u+(-\Delta)^{\delta} \partial_{t}^{2} u+\Delta^{2} u+a(-\Delta)^{\alpha} u+(-\Delta)^{\theta} \partial_{t} u=0 \\
u(0, x)=u_{0}(x), \quad \partial_{t} u(0, x)=u_{1}(x),
\end{array}\right.
$$

where $u=u(t, x)$, with $(t, x) \in] 0, \infty\left[\times \mathbb{R}^{n}\right.$ and $a>0$ is a constant. The exponents of Laplacian $\delta, \alpha, \theta$ are such that $0 \leq \delta \leq 2,0 \leq \alpha \leq 2$ and $0 \leq \theta \leq(2+\delta) / 2$.

To show the existence and uniqueness of the solution, we consider two cases depending on the exponents of the Laplacian operators,

$$
\text { (i) } 0 \leq \theta<\delta \text { and } 0 \leq \delta \leq 2 \quad \text { (ii) } 0 \leq \delta \leq \theta \text { and } 0 \leq \theta \leq \frac{2+\delta}{2} .
$$

and for each case we consider operators that reduced the problem to a first order one. In case (i) we have the operator $B_{1}: H^{4-\delta}\left(\mathbb{R}^{n}\right) \times H^{2}\left(\mathbb{R}^{n}\right) \rightarrow X$ and $J_{1}: X \rightarrow X$ are given by

$$
B_{1}=\left[\begin{array}{cc}
0 & I \\
-A_{2} & 0
\end{array}\right] \quad \text { and } \quad J_{1}(U)=\left[\begin{array}{c}
0 \\
\left(I+(-\Delta)^{\delta}\right)^{-1}\left(u-(-\Delta)^{\theta} v\right)
\end{array}\right]
$$

where the operator $A_{2}=\left(I+(-\Delta)^{\delta}\right)^{-1}\left(\alpha \Delta^{2}-\Delta+I\right)$ is defined in $H^{4-\delta}\left(\mathbb{R}^{n}\right)$. In the second case we consider the operators $B_{2}: H^{4-\delta}\left(\mathbb{R}^{n}\right) \times H^{2}\left(\mathbb{R}^{n}\right) \rightarrow X$ and $J_{2}: X \rightarrow X$ are given below,

$$
B_{2}=\left[\begin{array}{cc}
0 & I \\
-A_{2} & -A_{\theta}
\end{array}\right] \quad \text { and } \quad J_{2}(U)=\left[\begin{array}{c}
0 \\
\left(I+(-\Delta)^{\delta}\right)^{-1}(u+v)
\end{array}\right] .
$$


where $A_{\theta}=\left(I+(-\Delta)^{\delta}\right)^{-1}\left(I+(-\Delta)^{\theta}\right)$ defined in $D\left(A_{\theta}\right)=H^{2 \theta-\delta}\left(\mathbb{R}^{n}\right)$ e $A_{2}$ is the same as in case (i). form

Using the energy space $X=H^{2}(\mathbb{R}) \times H^{\delta}\left(\mathbb{R}^{n}\right)$ we can rewrite the problem (3) in matrix

$$
\left\{\begin{array}{l}
\frac{d U}{d t}=B U+J(U) \quad \text { for all } \quad t>0 \\
U(0)=U_{0}
\end{array}\right.
$$

where $U=\left(u, \partial_{t} u\right), U(0)=\left(u_{0}, u_{1}\right)$ and the operators $B$ and $J$ adequate for each case.

Using the Lumer Phillips Theorem in A. Pazzy [?], we are going to prove that $B$ is the infinitesimal generator of contraction semigroup of class $C_{0}$ in $X$ and that $J$ is a bounded operator in $X$. Then, $B+J$ generate a semigroup of $C_{0}$ class in $X$. That is, there exist unique global solution for the associated linear Cauchy Problem (3).

Theorem 2.1. Let $n \geq 1,0 \leq \delta \leq 2$ and $0 \leq \theta \leq(2+\delta) / 2$. If $u_{0} \in H^{4-\delta}\left(\mathbb{R}^{n}\right) e$ $u_{1} \in H^{2}\left(\mathbb{R}^{n}\right)$ then the Cauchy Problem (3) have only one solution $u$ in following class

$$
u \in C^{2}\left(\left[0, \infty\left[; H^{\delta}\left(\mathbb{R}^{n}\right)\right) \cap C^{1}\left(\left[0, \infty\left[; H^{2}\left(\mathbb{R}^{n}\right)\right) \cap C\left(\left[0, \infty\left[; H^{4-\delta}\left(\mathbb{R}^{n}\right)\right) .\right.\right.\right.\right.\right.\right.
$$

\section{Existence and Uniqueness: Semilinear Problem}

We consider the following Cauchy problem for the semilinear equation in $\mathbb{R}^{n}$

$$
\left\{\begin{array}{l}
\partial_{t}^{2} u+(-\Delta)^{\delta} \partial_{t}^{2} u+\Delta^{2} u+a(-\Delta)^{\alpha} u+(-\Delta)^{\theta} \partial_{t} u=\beta(-\Delta)^{\gamma} u^{p}, \\
u(0, x)=u_{0}(x), \quad \partial_{t} u(0, x)=u_{1}(x),
\end{array}\right.
$$

where $u=u(t, x)$, with $(t, x) \in] 0, \infty\left[\times \mathbb{R}^{n}, a>0, \beta \neq 0\right.$ and $p>1$ integer. The fractional powers of the Laplacian operator are considered as follows $0 \leq \delta \leq 2,0 \leq \alpha \leq 2$, $0 \leq \theta \leq(2+\delta) / 2$ and $1 / 2 \leq \gamma \leq(2+\delta) / 2$.

As in the linear problem, to study the existence of solutions, we need to divide the problem into two cases as in (4).

We reduce the order of the Cauchy Problem (5) and rewrite it in the following matrix form

$$
\left\{\begin{array}{l}
\frac{d U}{d t}=B U+F(U) \quad \text { for all } \quad t>0 \\
U(0)=U_{0}
\end{array}\right.
$$

where $U=\left(u, \partial_{t} u\right), U_{0}=\left(u_{0}, u_{1}\right)$ and the operator $B$ is defined in the Section 2 of according to each both cases mentioned above and is the infinitesimal generator of contraction of semigroup of class $C_{0}$ in $X$. The operator $F$ is the operator which contains the non-linear term.

\subsection{Local Existence}

Since $B$ is the infinitesimal generator of contractions semigroup of class $C_{0}$ in $X$, and if we prove that the operator $F$ is well defined as an operator $F: D(B) \rightarrow D(B)$ and is 
Lipschitz continuous on bounded sets, then we can apply the Theorem 1.1 to conclude that there exists unique solution $U$ in a maximal interval $\left[0, T_{m}\right.$ [, for each initial data $U_{0} \in D(B)$, such that one and only one of the following conditions below is true,

$$
\text { i) } T_{m}=\infty \quad \text { ii) } T_{m}<\infty \text { and } \lim _{t \rightarrow T_{m}}\left\{\|U\|_{X}+\|B U\|_{X}\right\}=\infty \text {. }
$$

In addition, we have $U \in C^{1}\left(\left[0, T_{m}[; X) \cap C\left(\left[0, T_{m}[; D(B))\right.\right.\right.\right.$.

As is the case (i) of (4) the local existence we must consider the fractional power $\gamma$ in the following interval $0 \leq \gamma \leq(2+\delta) / 2$. As in the linear case let us consider the usual energy space $X$. Consider the system (5) rewrite in matrix form

$$
\left\{\begin{array}{l}
\frac{d U}{d t}=B_{1} U+F_{1}(U) \\
U(0)=U_{0}
\end{array}\right.
$$

where $U=(u, v) \in X, U_{0}=\left(u_{0}, u_{1}\right) \in X$ and the operators $B_{1}: H^{4-\delta}\left(\mathbb{R}^{n}\right) \times H^{2}\left(\mathbb{R}^{n}\right) \rightarrow X$ and $F_{1}: D\left(B_{1}\right) \rightarrow D\left(B_{1}\right)$ are given by

$$
B_{1}=\left[\begin{array}{cc}
0 & I \\
-A_{2} & 0
\end{array}\right] \quad \text { and } \quad F_{1}(U)=\left[\left(\begin{array}{c}
0 \\
\left(I+(-\Delta)^{\delta}\right)^{-1}\left(u-(-\Delta)^{\theta} v+\beta(-\Delta)^{\gamma} u^{p}\right)
\end{array}\right] .\right.
$$

Thus, we have that $F_{1}$ is Lipschitz continuous on bounded sets of $D\left(B_{1}\right)$ and due to the fact that $B_{1}$ is the infinitesimal generator of contractions semigroup of class $C_{0}$ in $X$ by the Theorem 1.1, we get the following theorem of existence and uniqueness of solutions.

Theorem 3.1. Let $0 \leq \delta \leq \theta, 0 \leq \theta \leq(2+\delta) / 2,0 \leq \gamma \leq(2+\delta) / 2, p>1$ integer and $0<n<8-2 \delta$. Then, for all initial data $\left(u_{0}, u_{1}\right) \in H^{4-\delta}\left(\mathbb{R}^{n}\right) \times H^{2}\left(\mathbb{R}^{n}\right)$ there exist only one solution for semilinear Cauchy problem (5) in the maximal interval $\left[0, T_{m}[\right.$ in a class

$$
u \in C^{2}\left(\left[0, T_{m}\left[; H^{\delta}\left(\mathbb{R}^{n}\right)\right) \cap C^{1}\left(\left[0, T_{m}\left[; H^{2}\left(\mathbb{R}^{n}\right)\right) \cap C\left(\left[0, T_{m}\left[; H^{4-\delta}\left(\mathbb{R}^{n}\right)\right)\right.\right.\right.\right.\right.\right.
$$

with one and only one of following conditions true,

$$
\text { i) } T_{m}=\infty \quad \text { ii) } T_{m}<\infty \quad \text { and } \quad \lim _{t \rightarrow T_{m}}\left\{\|U\|_{X}+\left\|B_{1} U\right\|_{X}\right\}=\infty .
$$

In the second case (ii) in (4) rewriting the system (5) in the matrix form we have

$$
\left\{\begin{array}{l}
\frac{d U}{d t}=B_{2} U+F_{2}(U) \quad \text { for all } \quad t>0 \\
U(0)=U_{0}
\end{array}\right.
$$

where $U(t)=(u, v)$ and $U_{0}=\left(u_{0}, u_{1}\right)$ in $X$ and the operators $B_{2}: H^{4-\delta}\left(\mathbb{R}^{n}\right) \times H^{2}\left(\mathbb{R}^{n}\right) \rightarrow$ $X$ and $F_{2}: D\left(B_{2}\right) \rightarrow D\left(B_{2}\right)$ given by

$$
B_{2}=\left[\begin{array}{cc}
0 & I \\
-A_{2} & -A_{\theta}
\end{array}\right] \quad \text { and } \quad F_{2}(U)=\left[\begin{array}{c}
0 \\
\left(I+(-\Delta)^{\delta}\right)^{-1}\left(u+v+\beta(-\Delta)^{\gamma} u^{p}\right)
\end{array}\right] .
$$


In a similar way to the previous section we can prove, also in this case, that $F_{2}$ is well defined and is Lipschitz continuous on bounded sets of $D\left(B_{2}\right)$ and a $B_{2}$ is infinitesimal generator of a contractions semigroup of class $C_{0}$ in $X$. Then, using again the Theorem 1.1, we conclude the following theorem of existence and uniqueness of solutions.

Theorem 3.2. Let $0 \leq \theta<\delta, 0 \leq \delta \leq 2,0 \leq \gamma \leq(2+\delta) / 2, p>1$ integer and $0<n<8-4 \delta$. Then, for initial data $\left(u_{0}, u_{1}\right) \in H^{4-\delta}\left(\mathbb{R}^{n}\right) \times H^{2}\left(\mathbb{R}^{n}\right)$ there exist only one solution for semilinear Cauchy problem (5) defined in a maximal interval $\left[0, T_{m}[\right.$ in class

$$
u \in C^{2}\left(\left[0, T_{m}\left[; H^{\delta}\left(\mathbb{R}^{n}\right)\right) \cap C^{1}\left(\left[0, T_{m}\left[; H^{2}\left(\mathbb{R}^{n}\right)\right) \cap C\left(\left[0, T_{m}\left[; H^{4-\delta}\left(\mathbb{R}^{n}\right)\right)\right.\right.\right.\right.\right.\right.
$$

with one and only one of the following conditions true

$$
\text { i) } T_{m}=\infty \quad \text { ii) } T_{m}<\infty \quad \text { and } \quad \lim _{t \rightarrow T_{m}}\left\{\|U\|_{X}+\left\|B_{2} U\right\|_{X}\right\}=\infty
$$

\subsection{Global Existence}

In this section we show that the maximal interval of existence in the previous cases is $[0, \infty[$, by assuming the additional condition $\gamma \geq \alpha / 2$. For this, let us assume that $T_{m}<\infty$ and we will prove $\|U\|_{X}+\|B U\|_{X}<\infty$, so we conclude that $T_{m}=\infty$.

Applying the Fourier Transform in the variable $x$ in the Cauchy Problem (5) we find the following equivalent Cauchy problem in the Fourier space

$$
\left\{\begin{array}{l}
\left(1+|\xi|^{2 \delta}\right) \partial_{t}^{2} \widehat{u}+\left(|\xi|^{4}+a|\xi|^{2 \alpha}\right) \hat{u}+|\xi|^{2 \theta} \partial_{t} \widehat{u}=\beta|\xi|^{2 \gamma} \widehat{u^{p}} \\
\hat{u}(0, \xi)=\hat{u}_{0}(\xi), \quad \partial_{t} \widehat{u}(0, \xi)=\hat{u}_{1}(\xi) .
\end{array}\right.
$$

Using the principle of Duhamel, we know that the solution of the Cauchy Problem (6) is given by

$$
\hat{u}(t, \xi)=\hat{H}(t, \xi) \hat{u}_{0}+\hat{G}(t, \xi) \hat{u}_{1}+\beta \int_{0}^{t} \hat{G}(t-\tau, \xi) \frac{|\xi|^{2 \gamma}}{1+|\xi|^{2 \delta}} \widehat{u^{p}}(\tau, \xi) d \tau
$$

and we still have to

$$
\partial_{t} \widehat{u}(t, \xi)=\partial_{t} \widehat{H}(t, \xi) \hat{u}_{0}+\partial_{t} \widehat{G}(t, \xi) \hat{u}_{1}+\beta \int_{0}^{t} \partial_{t} \widehat{G}(t-\tau, \xi) \frac{|\xi|^{2 \gamma}}{1+|\xi|^{2 \delta}} \widehat{u^{p}}(\tau, \xi) d \tau,
$$

where

$$
\begin{aligned}
& \hat{G}(t, \xi)=\frac{e^{\lambda_{+} t}-e^{\lambda_{-} t}}{\lambda_{+}-\lambda_{-}} \quad \text { and } \quad G(t, x)=\mathfrak{F}^{-1}(\hat{G}(t, \cdot))(x), \\
& \hat{H}(t, \xi)=\frac{\lambda_{+} e^{\lambda_{-} t}-\lambda_{-} e^{\lambda_{+} t}}{\lambda_{+}-\lambda_{-}} \quad \text { and } \quad H(t, x)=\mathfrak{F}^{-1}(\hat{H}(t, \cdot))(x)
\end{aligned}
$$

being that the exponent $\lambda_{ \pm}$has the following form

$$
\lambda_{ \pm}=\frac{-|\xi|^{2 \theta} \pm \sqrt{|\xi|^{4 \theta}-4|\xi|^{2}\left(1+|\xi|^{2 \delta}\right)\left(|\xi|^{2}+a|\xi|^{2 \alpha-2}\right)}}{2\left(1+|\xi|^{2 \delta}\right)}
$$


The following estimates

$$
\begin{aligned}
\left(1+|\xi|^{2 \delta}\right)\left|\partial_{t} \widehat{u}\right|^{2}+|\xi|^{2} & \left(|\xi|^{2}+a|\xi|^{2 \alpha-2}\right)|\hat{u}|^{2} \\
& \leq 5 e^{-\frac{1}{5} \rho_{\theta}(\xi) t}\left(\left(1+|\xi|^{2 \delta}\right)\left|\hat{u}_{1}\right|^{2}+|\xi|^{2}\left(|\xi|^{2}+a|\xi|^{2 \alpha-2}\right)\left|\hat{u}_{0}\right|^{2}\right)
\end{aligned}
$$

can be proved in a similar way to estimate in the Lemma 3.6 of Horbach et al. [3], where

$$
\rho_{\theta}(\xi)=\left\{\begin{array}{llll}
\varepsilon|\xi|^{2-2 \theta}\left(|\xi|^{2}+a|\xi|^{2 \alpha-2}\right), & |\xi| \leq 1 & \text { e } & 0 \leq \theta \leq \frac{\alpha}{2} \\
\varepsilon \frac{|\xi|^{2 \theta}}{1+|\xi|^{2 \delta}}, & |\xi| \leq 1 & \text { e } & \frac{\alpha}{2}<\theta \leq \frac{2+\delta}{2} \\
\varepsilon \frac{|\xi|^{2 \theta}}{1+|\xi|^{2 \delta}}, & |\xi| \geq 1 & \text { e } & 0 \leq \theta \leq \frac{2+\delta}{2} .
\end{array}\right.
$$

Lemma 3.1. Let $\hat{G}(t, \xi)$ and $\hat{H}(t, \xi)$ fundamental solutions of linear problem associated to problem (6). Then we have the following estimates,
i) $\left|\partial_{t} \hat{G}\right|^{2} \leq 5 e^{-\frac{1}{5} \rho_{\theta}(\xi) t}$;
iii) $\quad\left|\partial_{t} \hat{H}\right|^{2} \leq 5 e^{-\frac{1}{5} \rho_{\theta}(\xi) t} \frac{|\xi|^{2}\left(|\xi|^{2}+a|\xi|^{2 \alpha-2}\right)}{\left(1+|\xi|^{2 \delta}\right)} ;$
ii) $\quad|\hat{G}|^{2} \leq 5 e^{-\frac{1}{5} \rho_{\theta}(\xi) t} \frac{\left(1+|\xi|^{2 \delta}\right)}{|\xi|^{2}\left(|\xi|^{2}+a|\xi|^{2 \alpha-2}\right)} ;$
iv) $|\hat{H}|^{2} \leq 5 e^{-\frac{1}{5} \rho_{\theta}(\xi) t}$.

The proof of the Lemma 3.1 uses the relation (9).

Again in case (i) of (4) the estimate of $\|U(t)\|_{X}^{2}+\left\|B_{1} U(t)\right\|_{X}^{2}$ for all $t \in\left[0, T_{m}[\right.$, with the assumption that $T=T_{m}<\infty$, where $U=\left(u, \partial_{t} u\right)$ and $u$ is the solution of (5) given by Theorem 3.2 .

In the process of proof we obtain the following inequality

$$
M_{1}(t) \leq C\left(\left\|B_{1} U_{0}\right\|_{X}^{2}+\left\|u_{1}\right\|_{\dot{W}_{-1,1}}^{2}\right)+C T_{m} M_{1}(t)^{p},
$$

for $t \in\left[0, T_{m}\left[\right.\right.$ with $T_{m}<\infty$ and $M_{1}(t) \doteqdot \sup _{0 \leq \tau \leq t}\left(\|U(\tau)\|_{X}^{2}+\left\|B_{1} U(\tau)\right\|_{X}^{2}\right)$, for $0 \leq t<T_{m}$.

To show that the solution of the Cauchy Problem (5) is global, that is, $T_{m}=+\infty$ we will need the following elementary lemma of calculus.

Lemma 3.2. Let $p>1$ and $F(M)=a I_{0}+b T M^{p}-M, M \geq 0$, a real continuous function, with $a, b, I_{0}, T$ positive constants and $M \geq 0$. Then, there exists unique $M_{0}>0$ absolute minimum point of $F(M)$ in $\left[0, \infty\left[\right.\right.$. In addition, there exists $\varepsilon>0$ such that if $0<I_{0} \leq \varepsilon$ then $F\left(M_{0}\right)<0$.

We note that the function $M_{1}(t)$ is not negative and satisfy a inequality $F\left(M_{1}(t)\right) \geq 0$ for all $t \in\left[0, T_{m}\right.$ [ due to inequality (10) with $F(M)$ the function given in Lemma 3.2 with $a=b=C, T=T_{m}$ and $I_{0}=\left\|B_{1} U_{0}\right\|_{X}^{2}+\left\|u_{1}\right\|_{\dot{W}_{-1,1}}^{2}$.

Therefore, if $0<I_{0} \leq \varepsilon$ and $\varepsilon>0$ given by Lemma 3.2, due to the continuity of the function $M_{1}(t)$, there are only two possibilities,
(a) $M_{1}(t)<M_{0}$ for all $t \in\left[0, T_{m}[\right.$
or
(b) $M_{1}(t)>M_{0}$ for all $t \in\left[0, T_{m}[\right.$. 
However, we note that $M_{1}(0)=\left\|U_{0}\right\|_{X}^{2}+\left\|B_{1} U_{0}\right\|_{X}^{2}$. Then, assuming another condition on the initial data that $M_{1}(0)<M_{0}\left(M_{0}\right.$ the global minimum point of Lemma 3.2), it follows that $M_{1}(t) \leq M_{0}$ for all $t \in\left[0, T_{m}\right.$ [ by the continuity of $M_{1}(t)$ with $t \in\left[0, T_{m}\right]$. The condition (a) holds.

Therefore, if $T_{m}$ is finite, it follows that $\|U\|_{X}^{2}+\left\|B_{1} U\right\|_{X}^{2}$ is also bounded for all $t \in\left[0, T_{m}\left[\right.\right.$. This contradicts the condition of Theorem 3.2. So, we must have $T_{m}=+\infty$ and the solution is global.

Theorem 3.3. Let $0 \leq \theta<\delta, 0 \leq \delta \leq 2,1 / 2 \leq \gamma \leq(2+\delta) / 2, p>1$ integer and $1 \leq n<8-2 \delta$. Consider the initial data $u_{0} \in H^{4-\delta}\left(\mathbb{R}^{n}\right)$ and $u_{1} \in H^{2}\left(\mathbb{R}^{n}\right) \cap \dot{W}_{-1,1}\left(\mathbb{R}^{n}\right)$ satisfying $0<I_{0} \leq \varepsilon$ and $M_{1}(0)<M_{0}$ with $\varepsilon, I_{0}, M_{0}, M_{1}(0)$ given above and in Lemma 3.2.

Then there exist only one global solution $u=u(t, x)$ for Cauchy Problem (5) such that

$$
u \in C^{2}\left(\left[0, \infty\left[; H^{\delta}\left(\mathbb{R}^{n}\right)\right) \cap C^{1}\left(\left[0, \infty\left[; H^{2}\left(\mathbb{R}^{n}\right)\right) \cap C\left(\left[0, \infty\left[; H^{4-\delta}\left(\mathbb{R}^{n}\right)\right) .\right.\right.\right.\right.\right.\right.
$$

In the second case (ii) of (4) it is shown that a limitation for $\|U\|_{X}^{2}+\left\|B_{2} U\right\|_{X}^{2}$ for all $t \in\left[0, T_{m}\right.$ [, with $U=\left(u, \partial_{t} u\right)$ and $u$ the solution of (5) given by Theorem 3.1. Analogously to the previous case one obtains the following theorem.

Theorem 3.4. Let $0 \leq \delta \leq \theta, 0 \leq \theta \leq(2+\delta) / 2,1 / 2 \leq \gamma \leq(2+\delta) / 2, p>1$ integer and $1 \leq n<8-2 \delta$. Consider the initial data $u_{0} \in H^{4-\delta}(\mathbb{R})$ and $u_{1} \in H^{2}\left(\mathbb{R}^{n}\right) \cap \dot{W}_{-1,1}\left(\mathbb{R}^{n}\right)$ satisfying $0<I_{0} \leq \varepsilon$ and $M_{2}(0)<M_{0}$ with $\varepsilon, I_{0}, M_{0}, M_{2}(0)$ given above and in Lemma 3.2.

Then there exist only one global solution $u=u(t, x)$ for Cauchy Problem (5) such that

$$
u \in C^{2}\left(\left[0, \infty\left[; H^{\delta}\left(\mathbb{R}^{n}\right)\right) \cap C^{1}\left(\left[0, \infty\left[; H^{2}\left(\mathbb{R}^{n}\right)\right) \cap C\left(\left[0, \infty\left[; H^{4-\delta}\left(\mathbb{R}^{n}\right)\right) .\right.\right.\right.\right.\right.\right.
$$

\section{References}

[1] C. I. Christov, G. A. Maugin \& M. G. Velarde, Well-posed Boussinesq paradigm with purely spatial higher-order derivatives, Physical Review E, vol. 54, no. 4 (1996): 3621-3638.

[2] A. Esfahani, L. G. Farah \& H. Wang, Global existence and blow-up for the generalized sixth-order Boussinesq equation, Nonlinear Analysis: Theory, Methods and Applications, vol. 75, no. 11 (2012): 4325-4338.

[3] J. L. Horbach, R. Ikehata, \& R. C. Charão, Optimal Decay Rates and Asymptotic Profile for the Plate Equation with Structural Damping, Journal of Mathematical Analysis and Applications, vol. 440, no. 2 (2016): 529-560.

[4] G. A. Maugin, Nonlinear Waves in Elastic Crystals, Oxford Science Publications, Oxford University Press, 1999.

[5] A. Pazy, Semigroups of linear operators and applications to partial differential equations, Springer-Verlag, New York, 1983.

[6] I. Segal, Non-Linear Semi-Groups. Annals of Mathematics, Second Series 78, no 2 (1963): 339-364. doi:10.2307/1970347 